\title{
THE INFLUENCE OF FREQUENCY-LIMITED AND NOISE-CONTAMINATED SENSING ON REACTIVE TURBULENCE CONTROL SCHEMES
}

\author{
Alexander Stroh ${ }^{1,2}$, Bettina Frohnapfel ${ }^{1,2}$, Yosuke Hasegawa ${ }^{2,4}$, \\ Nobuhide Kasagi ${ }^{4}$, Cameron Tropea ${ }^{1,2,3}$ \\ ${ }^{1}$ International Research Training Group Darmstadt-Tokyo on Mathematical Fluid Dynamics \\ ${ }^{2}$ Center of Smart Interfaces \\ ${ }^{3}$ Institute of Fluid Mechanics and Aerodynamics \\ Technische Universität Darmstadt, Germany \\ stroh/frohnapfel/tropea @csi.tu-darmstadt.de \\ ${ }^{4}$ Department of Mechanical Engineering \\ The University of Tokyo, Japan \\ hasegawa/kasagi @thtlab.t.u-tokyo.ac.jp
}

\begin{abstract}
Existing reactive turbulence control schemes for skin friction drag reduction are investigated to see how frequencylimited and noise-contaminated sensing will influence their control performance. It is found that the sensing frequency can be reduced substantially when the control scheme is modified to account for the convection speed of the measured sensor signal. Noisy sensor signals are shown to strongly affect the achievable net energy saving rate and energy gain at high sensing frequencies, while their influence is weaker for reduced sensing frequencies. These results also hold for increased Reynolds number where the performance of the control scheme is generally reduced.
\end{abstract}

\section{INTRODUCTION}

Turbulence control techniques leading to skin friction drag reduction are of great economical and ecological interest. In general, reactive control schemes exhibit considerable potential because of large energy gains that can be obtained with small power input. However, most previous numerical studies assume full spatio-temporal resolution of sensing and actuation over an entire wall, while real sensors and actuators have finite dimensions and limited frequency response. In order to transfer the existing control schemes to practical applications, a number of different limitations need to be considered. In the present work, we focus on the influences of sensors with limited frequency resolution and noise pollution on control performance.

Opposition control is a reactive control scheme where suction and blowing in the wall-normal direction or local spanwise wall velocity are introduced, so as to suppress the sweep and ejection events in the near wall region (Choi et al., 1994). The applied wall velocity is exactly opposite to the normal or spanwise component of the flow field at a prescribed sensing location, $y_{s}$, as sketched in figure 1 . The wall-normal opposition control is given by

$$
v(x, 0, z, t)=-\alpha v\left(x, y_{s}, z, t\right),
$$

whereas the spanwise opposition control by

$$
w(x, 0, z, t)=-\alpha w\left(x, y_{s}, z, t\right) .
$$

\section{PROCEDURE \\ Numerical methods}

The present investigation is performed using direct numerical simulations of a fully developed channel flow under a constant flow rate. The DNS-solver is based on a finite difference method executed on a staggered grid with a fractional step method for pressure decoupling. For temporal advancement, the convection terms and the viscous terms are discretized using the 2nd order Adams-Bashforth and the CrankNicholson method, respectively. All coordinates and velocity components in the present paper are normalized with the inner variables (plus units) of the uncontrolled flow at a given bulk Reynolds number. The computational time step is set to $\Delta t_{0}=0.03$ and total simulation time is given by $\Delta t=9000$. Two reference cases of uncontrolled channel flow are chosen at friction Reynolds number $\operatorname{Re}_{\tau}=u_{\tau} \delta / v=150$ and 300. The 


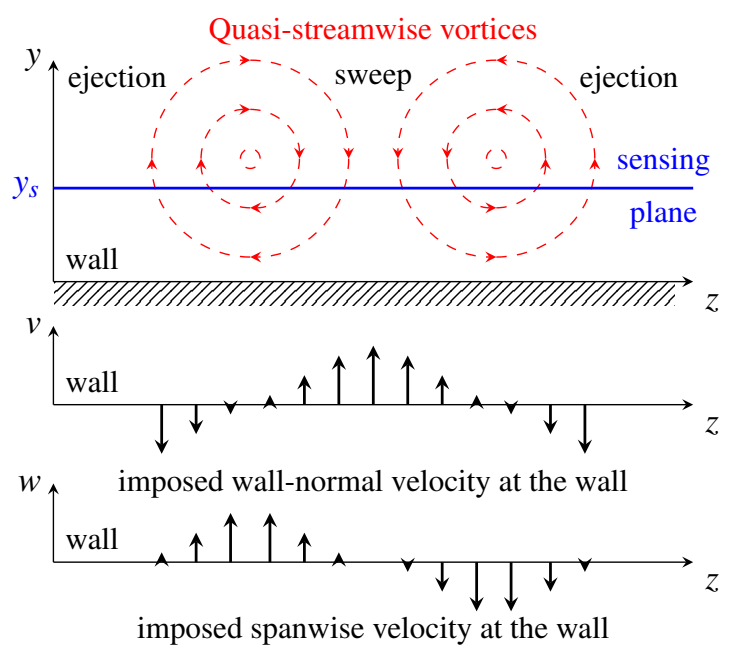

Figure 1. Schematic of near-wall quasi-streamwise vortices and applied control in wall-normal and spanwise opposition control.

corresponding bulk Reynolds numbers are $\operatorname{Re}_{b}=2 U_{b} \delta / v=$ 4560 and $R e_{b}=10110$, respectively. Detailed properties of the numerical domain for both cases are shown in table 1. It was confirmed that the obtained results are independent of grid resolution and domain size, and also that the uncontrolled reference cases are in good agreement with literature data (Kuroda et al., 1990).

Table 1. Grid resolution and domain size.

\begin{tabular}{ccccc}
\hline \hline $\operatorname{Re}_{\tau}$ & Grid size & Dimensions & $\Delta x$ & $\Delta z$ \\
\hline 150 & $64 \times 129 \times 64$ & $2.5 \pi \delta \times 2 \delta \times \pi \delta$ & 18.7 & 7.4 \\
300 & $160 \times 257 \times 128$ & $2 \pi \delta \times 2 \delta \times \pi \delta$ & 11.9 & 7.4 \\
\hline \hline
\end{tabular}

\section{Control performance}

In order to investigate the influence of frequency-limited and noise-contaminated sensing on reactive turbulence control schemes, we consider two opposition control schemes given by Choi et al. (1994); see equations (1) and (2). The control performance is evaluated following the suggestions of Kasagi et al. (2009) where the following performance indicators are introduced:

Drag reduction rate

$$
R=1-P / P_{0}
$$

Net energy saving rate

$$
S=1-\left(P+P_{\text {in }}\right) / P_{0}
$$

Energy gain

$$
G=\left(P_{0}-P\right) / P_{\text {in }}
$$

In these definitions the pumping power of the uncontrolled flow is denoted by $P_{0}$, while $P$ represents the pumping power of the controlled flow and $P_{i n}$ is the power input due to the applied control. For reactive flow control schemes with the goal of energy saving, the energy saving rate, $S$, basically replaces the drag reduction rate, $R$, which does not take into account the control power input, $P_{i n}$. Since energy recovery from the flow is unrealistic, we estimate $P_{i n}$ in the most conservative form by taking the absolute value of the local power input. Thereby the power input for wall-normal opposition control is defined by

$$
P_{i n, v}=\overline{\left(|p v|+\left|\frac{1}{2} v^{3}\right|\right)_{\text {wall }}},
$$

and for spanwise opposition control by

$$
P_{i n, w}=\overline{\left|w \frac{d w}{d y}\right|_{\text {wall }}},
$$

where the overbar denotes temporal and spatial averaging.

The performance of opposition control is strongly affected by the position of the sensing plane, $y_{s}$ (Choi et al., 1994). The highest drag reduction in the numerical experiments of Choi et al. (1994) with wall-normal and spanwise opposition control is achieved for $y_{s}=10$ at $\operatorname{Re}_{\tau}=180$. Later investigations of wall-normal opposition control show that the optimal sensing plane position is located at $y_{s}=15$ for the same Reynolds number (e.g. Hammond et al. (1998)). A variation of the sensing plane position in the present work yields the highest drag reduction at $y_{s}=15$ for wall-normal and at $y_{s}=10$ for spanwise opposition control at $\operatorname{Re}_{\tau}=150$. For both control schemes we choose the reference sensing plane position at $y_{s}=10$.

Another important parameter for opposition control is the amplitude of the imposed velocity at the wall, which can be described with an amplification factor, $\alpha$, introduced in equations (1) and (2). In a recent study, Chung \& Talha (2011) investigated the influence of $\alpha$ on the effectiveness of wallnormal opposition control. They show that $\alpha<1$ yields increased drag reduction if the sensing plane, $y_{s}$, is located above the optimum position, while $\alpha<1$ for sensing planes below the optimum decreases the control performance. In the present work, we consider opposition control schemes with constant $\alpha=1$ for all cases.

\section{Sensor noise}

In order to investigate the influence of sensor noise on the control performance, Gaussian white noise based on the equations of Fox et al. (1988) is introduced. The control input with additional sensor noise is modelled as:

$$
u_{i}(x, 0, z, t)=-u_{i}\left(x, y_{s}, z, t\right)+I \cdot n(x, z, t) \cdot u_{i, r m s}\left(y_{s}\right),
$$

where $I$ represents the noise intensity, $u_{i, r m s}\left(y_{s}\right)$ is the RMSvalue of the corresponding velocity component at the sensing plane, $y_{s}$, in uncontrolled flow and $n(x, z, t)$ is the generated noise which is spatially and temporally uncorrelated. 


\section{RESULTS AND DISCUSSION Influence of sensing frequency}

The maximum sensing and actuation frequency in the present numerical condition is given by $f_{0}=1 / \Delta t_{0}=33.33$. In order to investigate the influence of a limited frequency response of the sensors, we assume that sensing at $y_{s}$ is not carried out at every computational time step, but only with a certain sensing frequency $f_{s}=1 / \Delta t_{s}$, where $\Delta t_{s}$ is the time interval between discrete measurements at the sensor location. This is referred to a time-discrete control in contrast to a convected control introduced later.

Since the applied control at the wall depends on the sensor reading we also need to consider the actuation frequency $\Delta f_{a}=1 / \Delta t_{a}$. Initially, we adjust the actuation frequency to the sensing frequency, $f_{a}=f_{s}=f$. The obtained results for wall-normal opposition control operated with this timediscrete sensing and actuation are shown in figure 3 . The energy saving rate, $S$, decreases slightly down to $f \simeq 0.22$ and then drops rapidly while the gain, $G$, shows a continuous decrease.

At higher Reynolds number, $R e_{\tau}=300$, both, $S$ and $G$ are reduced. From the study of Iwamoto et al. (2002) it is known that wall-normal opposition control exhibits a strong Reynolds number effect at very low Reynolds numbers while it is almost insensitive to Reynolds number for $R e_{\tau}>300$. It can therefore probably be assumed that a further increase of the Reynolds number will not have a strong effect on the control performance. The Reynolds number dependency at low Reynolds numbers is mainly due to the increase of $P_{i n, v}$ caused by increasing pressure fluctuations at higher Reynolds number.

The lower limit of the sensing frequency is mainly determined by the temporal autocorrelation of the sensing quantity, $C\left(v\left(x, y_{s}, z, t\right), v\left(x, y_{s}, z, t+\Delta t\right)\right)$. Considering the spatiotemporal correlation, as shown in figure 2 , one finds that higher correlation values can be obtained if the sensing information is convected downstream with a convection velocity $U_{c}=\Delta x / \Delta t=10$. Since the spatio-temporal correlation remains almost unchanged for the controlled flow, this method can be used to decrease the sensing frequency down to $f_{s} \simeq 0.04$ with almost constant energy saving rate, as shown in figure 4 . The gain for this control scheme remains relatively large for low sensing frequencies. At $R e_{\tau}=300$ the decrease of $S$ and $G$ compared to $R e_{\tau}=150$ is similar to the one observed for the time-discrete control scheme in figure 3 .

In addition to the reduced sensing frequency, $f_{s}$, the actuation frequency, $f_{a}$, at which the control input at each actuation location is updated also influences the control performance. This effect is shown in figure 5: $S$ and $G$ both decrease with decreasing actuation frequency whereby the influence on $G$ is more pronounced.

The same methods of limited frequency resolution are tested for spanwise opposition control. The corresponding results are shown in figures 6 to 8 . Spanwise opposition control generally exhibits similar trends in terms of control performance with varying frequency resolution. However, it is less sensitive to a reduction of the sensing frequency, which can be reduced down to $f_{s} \simeq 0.08$ for the time-discrete control scheme (figure 6). The decrease of energy saving rate and gain for decreasing sensing frequencies occurs smoother than in the case of wall-normal control. The convected control extends the usable sensing frequency region down

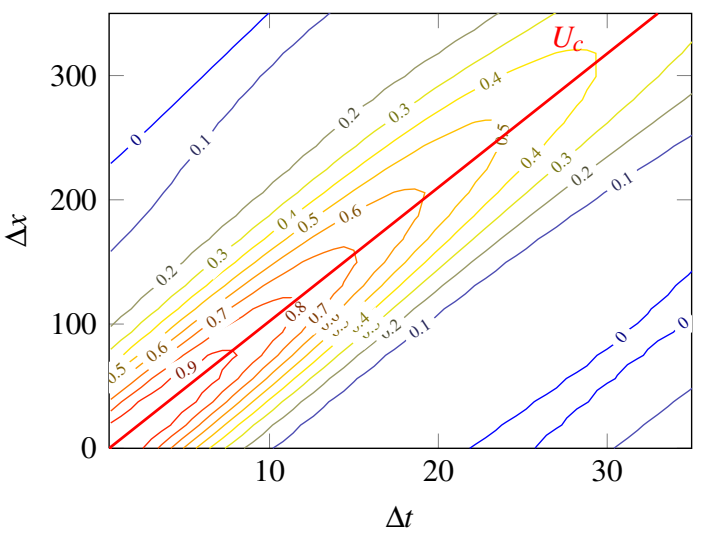

Figure 2. Spatio-temporal correlation of the sensing quantity, $C\left(v\left(x, y_{s}, z, t\right), v\left(x+\Delta x, y_{s}, z, t+\Delta t\right)\right)$.

to $f_{s} \simeq 0.02$ (figure 7 ). Furthermore, the spanwise convected scheme yields better results for decreasing actuation frequencies, i.e. $G=15$ for $f_{s} \simeq 0.06$ and $f_{a} \simeq 0.18$. At higher Reynolds number, the observed trends are similar to wallnormal opposition control, i.e. a decrease in $S$ and $G$ is observed. As for wall-normal opposition control, this decrease is mainly due to an increase of the power input. However, in spanwise opposition control, the power input, $P_{i n, w}$, does not depend on the pressure fluctuations but the Reynolds number dependency of the spanwise velocity fluctuations, $w$, can be assumed to be the major source for the performance decay. Generally, the influence of near-wall quasi-steamwise vortices on the flow field in the case of $\operatorname{Re}_{\tau}=300$ is excelled by the turbulent structures appearing above $y_{s}=30$, what makes the opposition control with sensing plane at $y_{s}=10$ less efficient in terms of $G$ (Iwamoto et al., 2002).

In respect to the influence of limited sensor frequency response, we conclude that opposition control can still be carried out for sensing frequencies down to $f_{s} \simeq 0.04$ when the newly introduced convected control scheme is applied. However, this is realized at the expense of a reduced energy gain compared to the original control scheme. A limited actuation frequency will further decrease the control performance. In general, spanwise opposition control turns out to be slightly less sensitive to the influence of limited frequency resolution than its wall-normal counterpart.

\section{Influence of noise contamination}

In order to investigate the influence of sensor noise, we carry out a parametric study varying the noise intensity, $I$, and the sensing frequency, $f_{s}$, for time-discrete and convected opposition control. At $R e_{\tau}=150$, the reference noise values, i.e. $u_{i, r m s}\left(y_{s}\right)$ in equation (8), are given by $v_{r m s}\left(y_{s}\right)=0.2772$ for wall-normal and $w_{r m s}\left(y_{s}\right)=0.7447$ for spanwise control schemes, respectively.

The results of the parametric study for time-discrete wall-normal opposition control are shown in figure 9. At high sensing frequencies, $G$ is significantly reduced even for low noise intensities due to the increase of pressure fluctuations caused by ,erroneous" suction and blowing. For the maximum sensing frequency, $f_{s}=33.33$, wall-normal control yields $G>1$ only for noise intensities up to $7-8 \%$. Interestingly, 
the control scheme becomes less sensitive to noise for lower sensing frequencies, allowing e.g. noise intensities up to $30 \%$ with $G>10$ and energy saving rate of $S \approx 20 \%$ for a sensing frequency of $f_{s} \simeq 0.26$. This increased noise resistance of the control scheme at lower sensing frequencies is due to the fact that noisy sensor signals introduce additional pressure fluctuations which enhance the power input, $P_{i n, v}$. If additional noise is introduced to the control system at a high frequency, $P_{i n, v}$ will increase drastically resulting in reduction of $S$ and $G$. It should be noted that the drag reduction rate, $R$, is not influenced by noisy sensor signals as long as the sensing frequency is significantly faster than the characteristic time scales of the flow (which can be determined from the energy spectrum of the flow). If noise is applied at lower frequencies, it modifies the flow field such that the effect on the control performance is found for all performance indicators, including $R$.

In the case of convected wall-normal opposition control, higher noise levels can be tolerated as shown in figure 10 . This scheme yields energy saving rates above $20 \%$ and gain of $10-15$ for sensing frequencies down to 0.05 and noise intensities up to $40 \%$. This reduced sensitivity to noisy sensor signals is due to the fact that the actuator input in the convected scheme is obtained by interpolation of different measurements which smoothes out the instantaneous fluctuations of the random noise and thus reduces the resulting pressure fluctuations.

The influence of noise-contaminated sensor signals on spanwise opposition control is shown in figures 11 and 12. In the case of time-discrete sensing and actuation (figure 11), the influence of noise on the control performance is almost independent of the sensing frequency in the range of $0.53<f_{s}<$ 33.33 , where positive energy saving rates are found for noise intensities up to $90-100 \%$. In contrast to wall-normal opposition control, the power input for spanwise opposition control, $P_{i n, w}$, is not governed by pressure fluctuations but only by the instantaneous spanwise velocity fluctuations and their wall-normal gradient (see equation 7). These fluctuations are naturally increased with increasing noise levels, but their dependence on the sensing frequency only becomes apparent for low sensing frequencies where the erroneous input at the actuator will lead to a deterioration of drag reduction itself indicating that the control principle does not work properly any more.

Similar to wall-normal control the application of the convected control scheme also increases the resistance to noisecontaminated sensor signals in spanwise opposition control (figure 12). The smoothing effect of the interpolation in the convected control scheme, which basically reduces the level of the introduced noise at the actuator, is more pronounced at lower sensing frequencies. The best results for $S$ and $G$ are obtained for sensor frequencies of $0.2<f_{s}<1$, yielding positive values of $S$ and $G>1$ for noise intensities up to $200 \%$.

\section{CONCLUSION AND OUTLOOK}

We investigate the effect of limited frequency resolution and sensor noise pollution in reactive control loops. These are two of the limitations that we face when considering the transfer of these idealized schemes to practical applications. It is found that larger sensing intervals can be realized with a convected control scheme. In this scheme the measured sensor signal is not used as constant actuator input until the next sensor signal is obtained, but instead, the sensor information is passed downstream with a convection velocity obtained from the spatio-temporal autocorrelation of the sensor signal.

In respect to noise-contaminated sensor signals, it is found that high frequency noise does not affect the drag reduction rate, but affects the control power input, and therefore the gain and the net energy saving rate significantly. For lower sensing frequencies, the sensor noise affects drag reduction directly, since the noise time-scales become close to those of the flow. In respect to Reynolds number dependency the trend reported by (Iwamoto et al., 2002) is confirmed, indicating a performance decrease of the control scheme with increasing Reynolds number. The observed trends in terms of frequencylimitation and noise-contamination do not exhibit a pronounced Reynolds number dependency.

In general, it can be concluded that a reduced sensing frequency for reactive turbulence control schemes with noisecontaminated sensor signals is beneficial in terms of control performance, especially if a control scheme is realized in which the sensor information is convected downstream over a number of actuators.

\section{ACKNOWLEDGEMENTS}

The authors greatly acknowledge the support by Deutsche Forschungsgemeinschaft, Japan Society for the Promotion of Science and MEXT Japan through projects IRTG 1529, FR2823/2-1, Grand-in-Aid for Scientific Research (A) (No. 20246036) and JSPS Postdoctoral Fellowship for Research Abroad (YH).

\section{LITERATURE}

Choi, H., Moin, P. \& Kim, J. 1994 Active turbulence control for drag reduction in wall-bounded flows. Journal of Fluid Mechanics 262, 75-110.

Chung, Y. M. \& Talha, T. 2011 Effectiveness of active flow control for turbulent skin friction drag reduction. Physics of Fluids 23.

Fox, R. F., Gatland, I. R., Roy, R. \& Vemuri, G. 1988 Fast, accurate algorithm for numerical simulation of exponentially correlated colored noise. Physical Review 38 (10), 59385940.

Hammond, E. P., Bewley, T. R. \& Moin, P. 1998 Observed mechanisms for turbulence attenuation and enhancement in opposition-controlled wall-bounded flows. Physics of Fluids 10, 2421.

Iwamoto, K, Suzuki, Y. \& Kasagi, N. 2002 Reynolds number effect on wall turbulence: Toward effective feedback control. International Journal of Heat and Fluid Flow 23, 678-689.

Kasagi, N., Hasegawa, Y. \& Fukagata, K. 2009 Toward costeffective control of wall turbulence for skin friction drag reduction. In Proceedings of the 12th EUROMECH European Turbulence Conference (ed. Bruno Eckhardt), pp. 189-200. Marburg, Germany: Springer.

Kuroda, A., Kasagi, N. \& Hirata, M. 1990 A direct numerical simulation of the turbulent flow between two parallel walls: Turbulence characteristics near the wall without mean shear. In 5th Symposium on Numerical Simulation ofTurbulence, pp. 1-5. IIS of the University of Tokyo. 

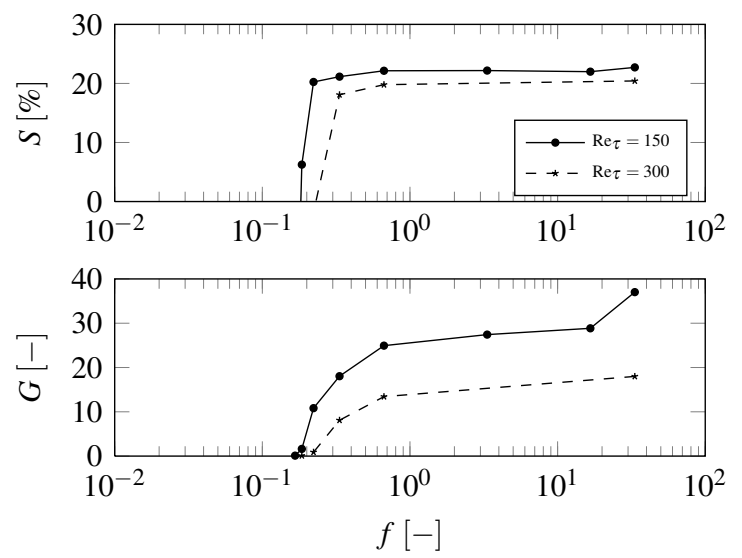

Figure 3. Performance indicators $S$ and $G$ for timediscrete wall-normal opposition control with varying frequency resolution of the sensor at $\operatorname{Re}_{\tau}=150$ and $\operatorname{Re}_{\tau}=300$.
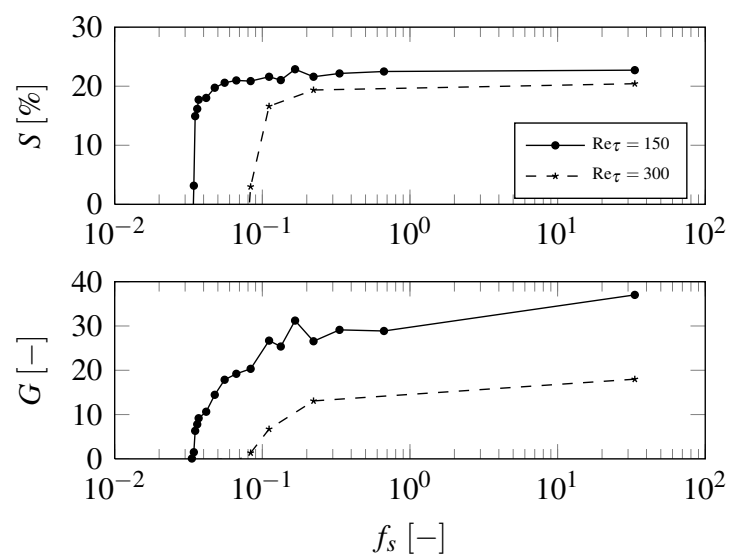

Figure 4. Performance indicators $S$ and $G$ for convected wall-normal opposition control with varying frequency resolution of the sensor at $\operatorname{Re}_{\tau}=150$ and $\operatorname{Re}_{\tau}=300$.
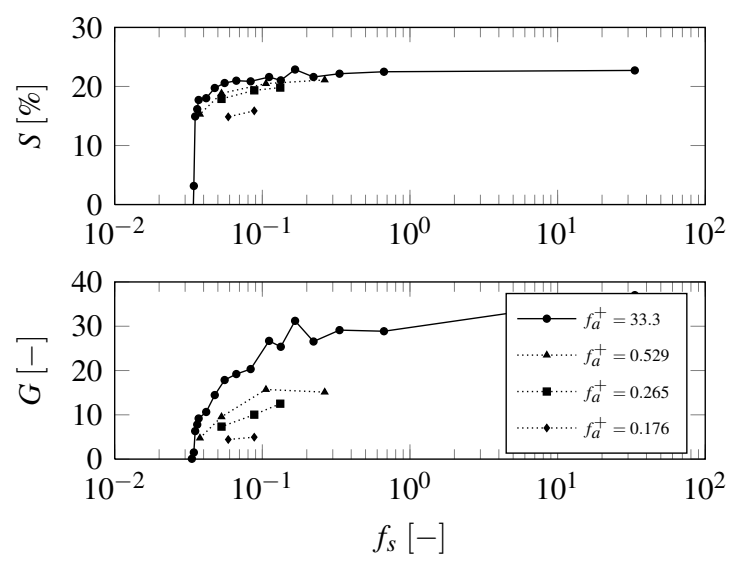

Figure 5. Performance indicators $S$ and $G$ for convected wall-normal opposition control with varying frequency resolution of sensor and actuator at $\operatorname{Re}_{\tau}=150$.
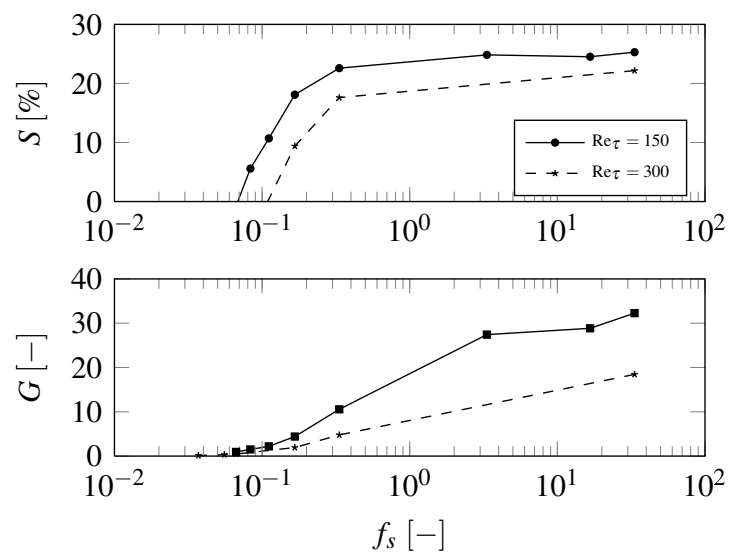

Figure 6. Performance indicators $S$ and $G$ for timediscrete spanwise opposition control with varying frequency resolution of the sensor at $\operatorname{Re}_{\tau}=150$ and $\operatorname{Re}_{\tau}=300$.
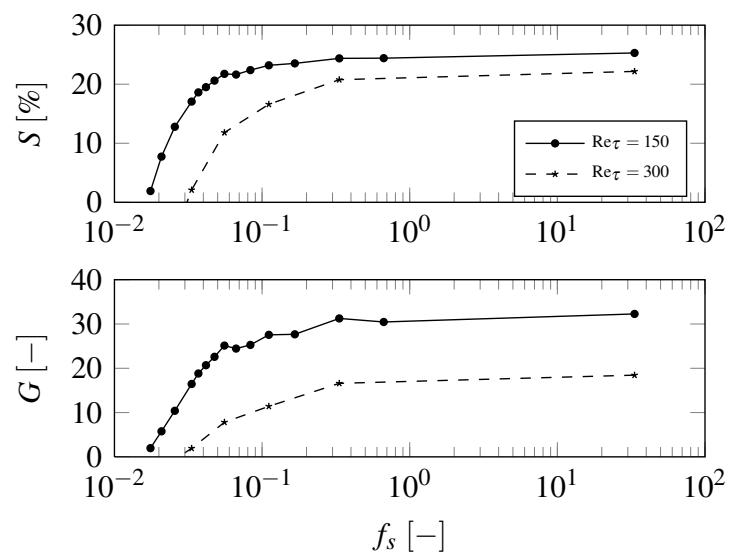

Figure 7. Performance indicators $S$ and $G$ for convected spanwise opposition control with varying frequency resolution of the sensor at $\operatorname{Re}_{\tau}=150$ and $\operatorname{Re}_{\tau}=300$.
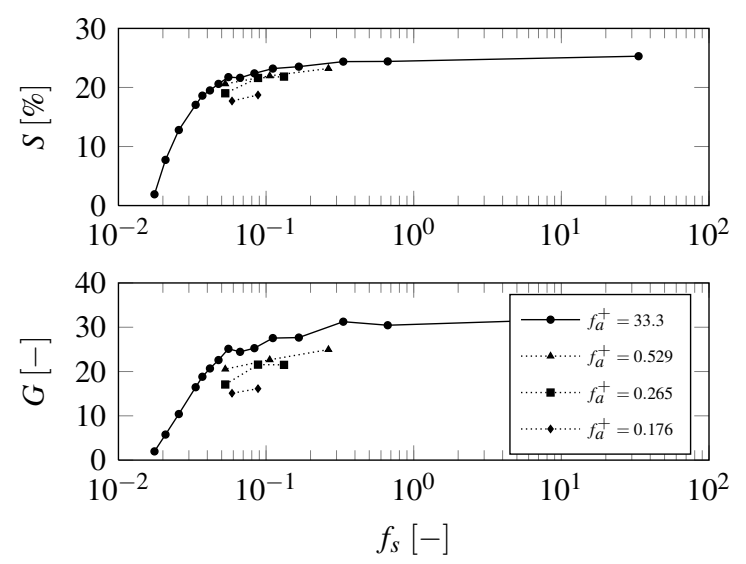

Figure 8. Performance indicators $S$ and $G$ for convected spanwise opposition control with varying frequency resolution of sensor and actuator at $\operatorname{Re}_{\tau}=150$. 

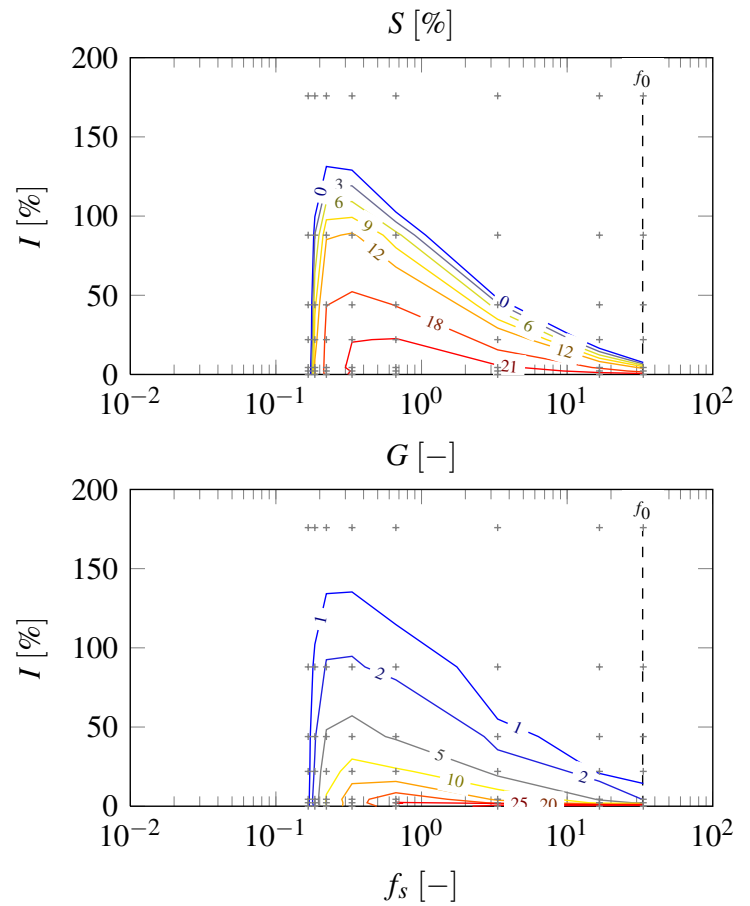

Figure 9. Performance indicators $S$ and $G$ for noise contaminated time-discrete wall-normal opposition control with varying frequency resolution of the sensor and varying noise intensity at $\operatorname{Re}_{\tau}=150$.
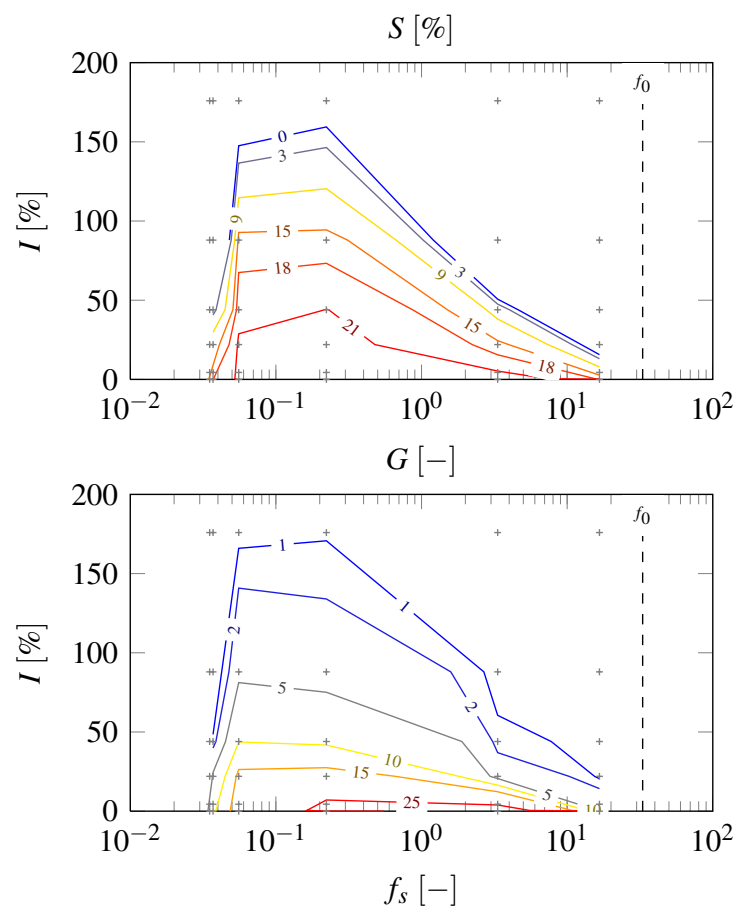

Figure 10. Performance indicators $S$ and $G$ for noise contaminated convected wall-normal opposition control with varying frequency resolution of the sensor and varying noise intensity at $\operatorname{Re}_{\tau}=150$.
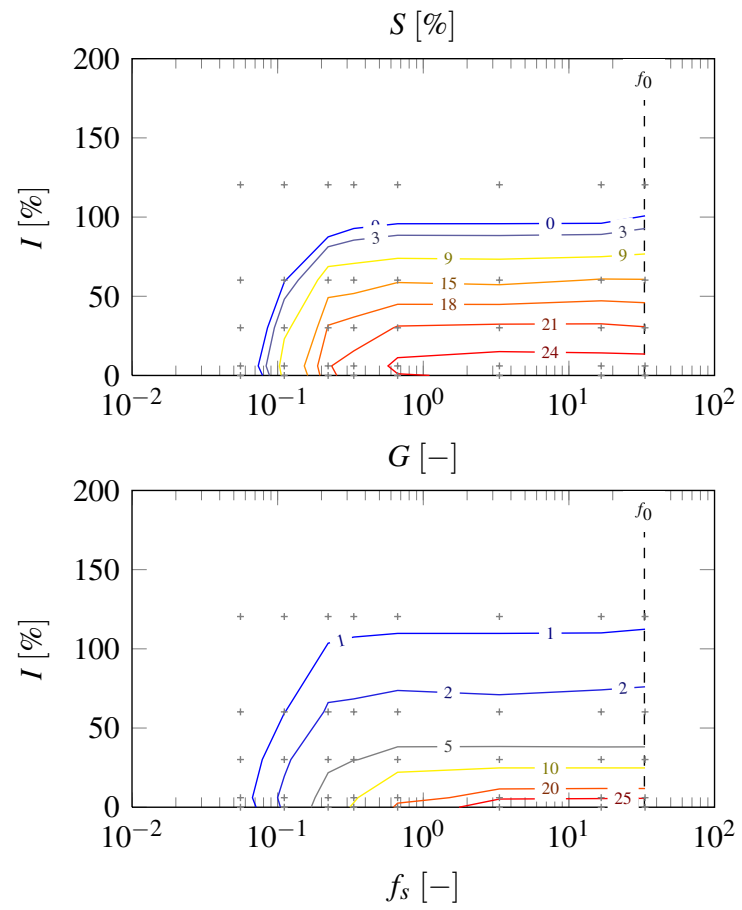

Figure 11. Performance indicators $S$ and $G$ for noise contaminated time-discrete spanwise opposition control with varying frequency resolution of the sensor and varying noise intensity at $\operatorname{Re}_{\tau}=150$.
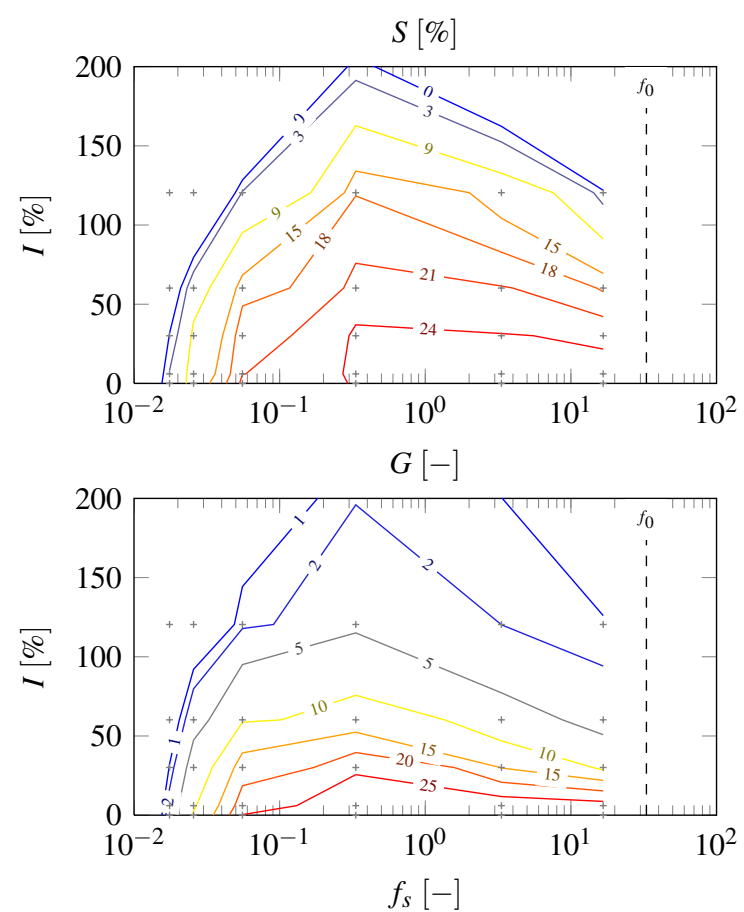

Figure 12. Performance indicators $S$ and $G$ for noise contaminated convected spanwise opposition control with varying frequency resolution of the sensor and varying noise intensity at $\operatorname{Re}_{\tau}=150$. 